\title{
License Plate Recognition System based on Improved BP Neural Network
}

\author{
Tingyu $\mathrm{Ma}$ \\ School of Electrical \\ Engineering, Jinan University, \\ Zhuhai 519070, PR China
}

\author{
Tao Wang \\ School of Electrical \\ Engineering, Jinan University, \\ Zhuhai 519070, PR China
}

\author{
Jinchao Shi \\ School of Electrical \\ Engineering, Jinan University, \\ Zhuhai 519070, PR China
}

\begin{abstract}
With the increase in the number of vehicles, the license plate recognition system has gradually become a hot spot in scientific research. In this paper, we address the shortcomings of BP neural networks and combine it with the image processing technology of license plate characters, propose a license plate recognition system based on improved BP neural network for license plate recognition. The system mainly includes four modules: license plate image preprocessing, license plate positioning, character segmentation and classification recognition. Firstly, image preprocessing is implemented through image graying, gray stretching, and image binarization. Secondly, edge detection and morphological processing are used to determine the license plate area. Thirdly, the character string of the license plate is segmented. Finally, two corresponding BP neural networks are designed to classify and recognize the license plate characters based on the characteristics of the license plates in China to obtain the recognition results.
\end{abstract}

\section{General Terms}

Image preprocessing, BP Neural Network

\section{Keywords}

LPR, License plate localization, Character segmentation, Classification recognition

\section{INTRODUCTION}

With the improvement of people's living standards and the rapid development of transportation and artificial intelligence, there are more and more vehicles and more and more traffic; At the same time, many scholars have begun to focus on the application of artificial intelligence technology in the transportation industry. The license plate recognition technology is an important part of the intelligent transportation system, and is widely used in various fields, such as toll systems, traffic monitoring, parking lot management, and so on. Effective license plate recognition technology can monitor vehicle violations, improve traffic management efficiency, and ensure social safety.

The basis for ensuring the correct recognition of license plates is the accurate positioning of license plates. At present, there are many methods for detecting and locating license plates. For example, a fast and efficient license plate location algorithm based on color and texture information. Through the analysis and research of each color component in the HSV space of the picture, the inherent characteristics of the license plate color are used to obtain the dynamic color threshold by calculating the color information of the entire image, the algorithm can accurately remove the complex background information in the license plate picture[1]. The vehicle is detected by the background difference method, and the license plate area is located by combining texture features and color features, respectively [2]. But there are some shortcomings, such as the execution speed is too slow.

In terms of license plate recognition, there are existing methods: A license plate recognition algorithm is designed based on character feature vectors and particle swarm optimization. The algorithm uses the vertical projection method, adaptive threshold scheme, direction gradient histogram method, etc. to segment the license plate characters and extract the feature vectors of the characters. The character recognition accuracy model is established based on the character feature vector samples and support vector machine, and the license plate recognition algorithm for solving the model is established based on the particle swarm optimization algorithm [3]. However, its calculation amount is large, and its license plate recognition ability is greatly affected by the training samples. A 13-point feature extraction algorithm was used to extract features for letters, numbers and Chinese characters and a three-layer BP neural network was constructed for recognition [4]. But BP neural network still has many shortcomings

In view of the shortcomings of the BP neural network, we have designed a vehicle license plate recognition system based on the improved BP neural network. After image preprocessing, license plate positioning, character segmentation, and classification recognition, the image signal is converted into a digital signal. By applying the GUI interface, the license plate recognition process is more intuitive, and the purpose of making the license plate recognition system more practical and convenient is achieved. The model is implemented in MATLAB development environment, with short training time and high recognition accuracy[6].

\section{BP NEURAL NETWORK}

A neural network is a simple model that abstracts the neuron system of the human brain. Interconnected neurons respond to external input signals to process information data. Constantly adjusting the weights of neurons can adapt to the application needs of different scenarios and achieve the best results. At present, there are various learning algorithms, the most widely used of which is the back-propagation algorithm. BP neural network refers to a multi-layer forward neural network based on the error back-propagation algorithm. Its neurons usually use the Sigmoid function to enable it to achieve non-linear mapping of input and output, so it has a wide range of applications in pattern recognition and other fields.

BP network includes input layer, hidden layer and output layer, and the neurons between adjacent layers are fully connected. Input is $X_{0}, X_{1}, X_{2}, \ldots, X_{n-1}$, output is $Y_{0}, Y_{1}, Y_{2}, \ldots, Y_{m-1}$. The expected output of the training sample 
is $d_{0}, d_{1}, d_{2}, \ldots, d_{n-1}$. The weight from input unit $i$ to intermediate unit $j$ is $V_{i j}$, the weight of the intermediate unit $j$ to the output unit $k$ is $W_{j k}$.

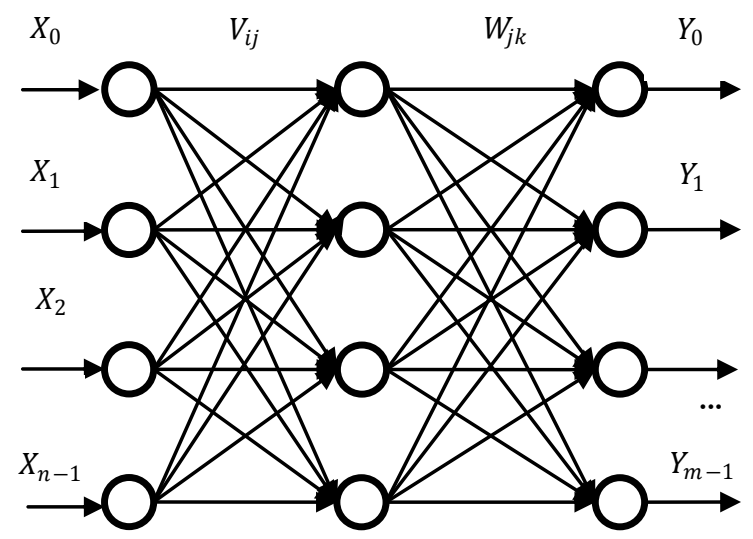

Fig 1: Schematic diagram of neural network structure The output of each unit in the middle layer:

$$
h_{j}=\sum_{i=0}^{N-1} V_{i j}-\Psi_{j}
$$

The output of each unit of the output layer:

$$
y_{k}=f\left[\sum_{j=0}^{L-1} V_{i j}-\Psi_{j}\right]
$$

The basic BP algorithm includes two processes: forward propagation of signals and back propagation of errors. The error output is calculated in the direction from input to output, and the weight and threshold are adjusted in the direction from output to input. During forward propagation, the input signal acts on the output node through the hidden layer. After the non-linear transformation, the output signal is generated. If the actual output does not match the expected output, the error is transferred to the backward propagation process of the error. Error back propagation is to transmit the output error layer by layer through the hidden layer to the input layer, and distribute the error to all units in each layer. The error signal obtained from each layer is used as the basis for adjusting the weight of each unit. By adjusting the connection strength of the input node and the hidden layer node, the connection strength of the hidden layer node and the output node, and the threshold value, the error is reduced in the gradient direction. After repeated learning and training, the network parameters (weights and thresholds) corresponding to the minimum error are determined, and the training stops. At this time, the trained neural network can process the input information of similar samples by itself and process the non-linearly transformed information with the smallest output error.

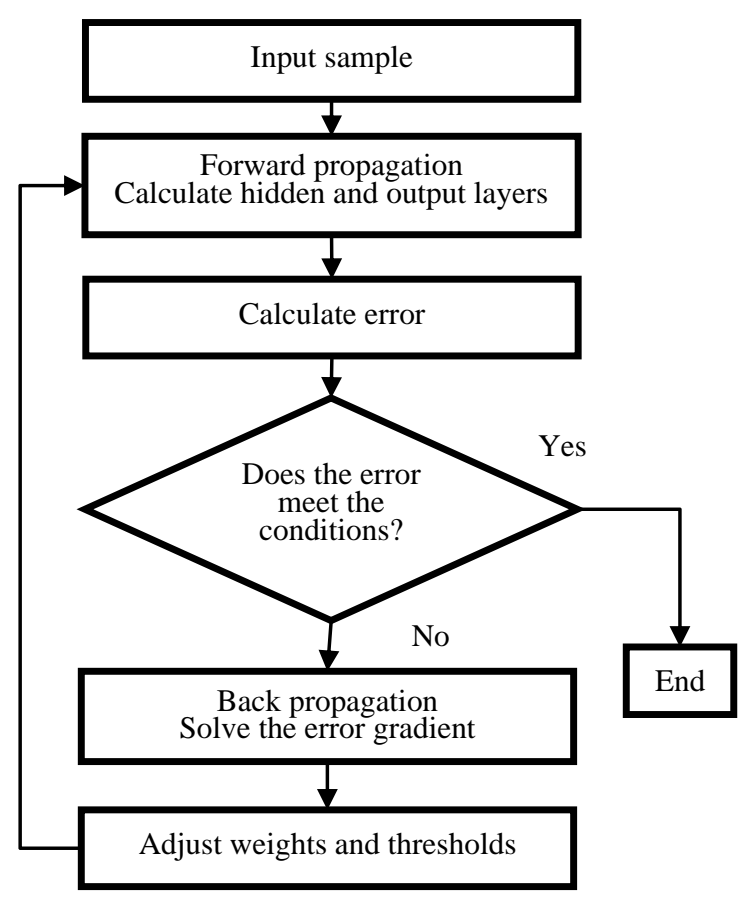

Fig 2: BP algorithm flowchart

We use BP neural network, through training the sample data, the network weights and thresholds are continuously modified to make the error adjustment direction always follow the direction in which the error decreases the fastest, thereby approaching the expected output.

\section{IMPROVEMENT OF BP NEURAL NETWORK}

\subsection{Deficiencies of BP Neural Network}

As the most widely used artificial neural network, BP neural network has many advantages, but there are still some shortcomings. First of all, the nonlinear optimization of BP neural network is prone to some minima problems, which has a negative impact on the network. In addition, when adjusting the weight, it does not take into account the gradient direction before time $t$, it just adjusts according to the time drop direction of the gradient error of $t$, so that the weight adjustment during the training process oscillates and the convergence speed is slow.

\subsection{Improved Scheme of BP Neural Network}

\subsubsection{Additional momentum}

The additional momentum factor algorithm refers to the addition of a change value that is proportional to the previous weight value on the basis of each weight change in the backpropagation process[15], Finally, a new change weight is generated according to the back-propagation principle. A weight adjustment with an additional momentum factor $\alpha(0<\alpha<1)$ is mainly used. The formula is:

$$
\begin{gathered}
\Delta \omega(k+1)=\alpha \Delta \omega(k)+(1-\alpha) \eta \frac{\partial E(k)}{\partial \omega(k)} \\
\omega(k+1)=\omega(k)+\Delta \omega(k+1)
\end{gathered}
$$

Where $\mathrm{k}$ is the number of trainings, and the following judgment conditions are used for additional momentum during training: 


$$
\alpha=\left\{\begin{array}{cc}
0 ; & \operatorname{SSE}(k)>1.05 \operatorname{SSE}(k-1) \\
0.95 ; & \operatorname{SSE}<\operatorname{SSE}(k-1) \\
\alpha ; & \text { others }
\end{array}\right.
$$

\subsubsection{Adaptive learning rate}

Adaptive learning rate refers to the automatic adjustment of the learning rate during the training process[1]. The adjustment formula is as follows:

$$
\eta(k+1)=\left\{\begin{array}{lr}
1.05 \eta(k) ; & \operatorname{SSE}(k+1)<\operatorname{SSE}(k) \\
0.7 \eta(k) ; & \operatorname{SSE}(k+1)>1.05 \operatorname{SSE}(k) \\
\eta(k) ; & \text { others }
\end{array}\right.
$$

The initial learning rate is $\eta(0)$, and its value can be chosen arbitrarily. SSE $(k)$ is the sum of squares of the kth error. The learning rate $\eta$ is also called the step size, which is a constant in the traditional BP algorithm. However, in actual calculations, it is difficult to give an optimal learning rate that is suitable from beginning to end. The adaptive adjustment of the learning rate $\eta$ algorithm can make it better for computing. The adjustment criterion is: if the correction value of the weight value reduces the error function, it means that the selected learning rate value is small, it can be increased by an amount; otherwise it is decreased.

\section{LICENSE PLATE RECOGNITION BASED ON BP NEURAL NETWORK}

The overall process of the license plate recognition system is shown in Figure 3. This system consists of four modules: image preprocessing, license plate positioning, character segmentation and classification recognition. The input of the system is the original RGB image collected through a camera or other means, and the output is the identification of the license plate information on the original image[8].

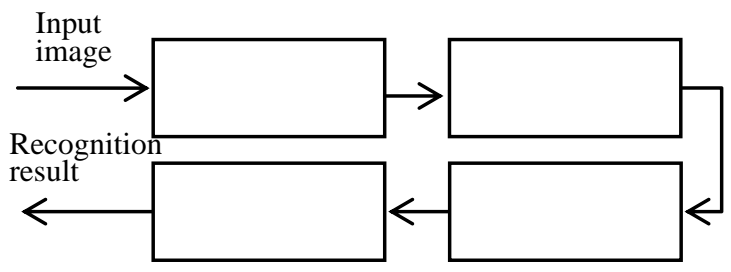

Fig 3: System flow chart

\subsection{Image Preprocessing}

When photographing, the formation or transmission of the image is affected by a variety of factors, such as distortion of the optical system, system noise, underexposure or overexposure, relative motion, etc. There is often a difference between the image and the original image. This difference is called degradation or degradation. Therefore, preprocessing must be performed before image processing, including removing noise, border enhancement, increasing brightness, and so on. The input color image contains a large amount of color information, which will take up more storage space, and will also slow down the system's execution speed during processing. Therefore, when processing the image, the color image is often converted into a grayscale image to speed up the processing. The main purpose of vehicle image preprocessing is to improve the quality of the vehicle image, especially the license plate area, while retaining and enhancing the texture and color information in the license plate, removing noise that may affect the texture and color information of the license plate area, and providing license plate positioning convenience.

\subsubsection{Image graying}

Due to the unevenness of light, camera, sensor sensitivity, and optical system, some parts of the image are darker or brighter, so the image needs to be grayed. Grayscale images do not contain color information, only brightness information. Generally, the image color includes three primary colors: R, $\mathrm{G}$, and $\mathrm{B}$. The process of setting the three primary color components equal is called the graying process. The image becomes a monochrome image with multiple gray values. Pixels with large gray values are lit, otherwise pixels are dark. There are three main methods for image graying:

a. Maximum value method: The value of $R, G$, and $B$ is equal to the largest of the three, and the formula is:

$$
R=G=B=\max (R, G, B)
$$

b. Average numerical method: R, G, and B after conversion are equal to the average of the three before conversion. The formula is:

$$
R=G=B=\frac{(R+G+B)}{3}
$$

c. Weighted average method: According to different importance, the corresponding weights of R, G, and $\mathrm{B}$ are assigned so that they are equal to the average of the weighted sum, respectively. The formula is:

$$
R=G=B=\frac{\left(R * P_{R}+G * P_{G}+B * P_{B}\right)}{3}
$$

Where, $P_{R}, P_{G}$ and $P_{B}$ are the weights of $\mathrm{R}, \mathrm{G}$, and $\mathrm{B}$, respectively.

The image before and after the graying process is shown in the figure:

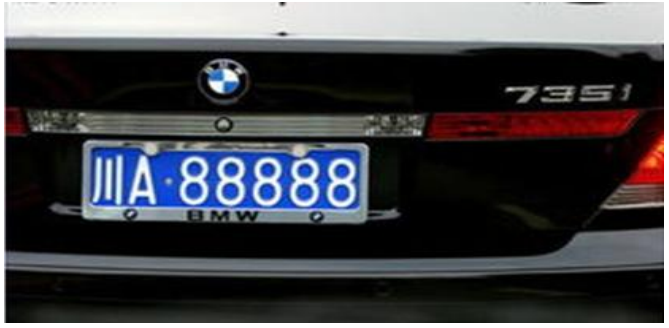

Fig 4: The original image

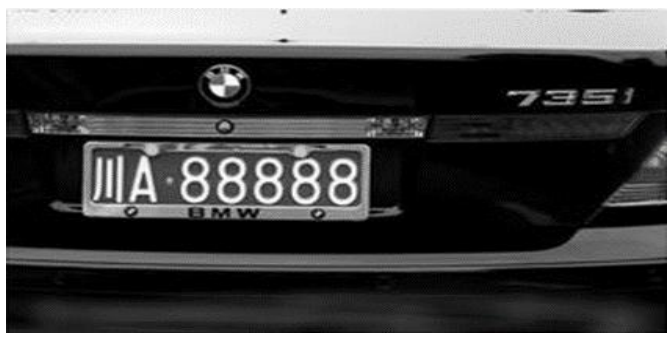

Fig 5: The grayscale image

\subsubsection{Gray scale stretching}

In the actual shooting environment, the scene is a threedimensional picture from far and near, and after shooting as an image, it is only a two-dimensional picture, which lacks the depth of the image. Therefore, the distant scenes and the near scenes will have a grayscale imbalance in the performance of the screen. Or the image is distorted due to the difference in sensitivity of the shooting device during the scanning process, 
and the gray range of the image is small due to the difference in exposure. The resulting image is a single gray level and blurred image. Therefore, a gray scale stretching operation is required.

Gray scale stretching is also called contrast stretching. It is the most basic type of gray scale transformation. It uses the simplest piecewise linear transformation function. Its main idea is to improve the dynamic range of gray scale during image processing. Image grayscale stretching is a method to change the contrast of an image. Through grayscale mapping, the grayscale value in a section of the original image is mapped to another grayscale value, thereby stretching or compressing the grayscale of the entire image. Distribution range, improving output image quality and enhancing contrast. The gray level $f(x, y)$ of a point $(x, y)$ in the original image is converted into the output image gray level $g(x, y)$ by a mapping function, the formula is as follows:

$$
g(x, y)=[(c+d) /(a+b)] f(x, y)+a
$$

\subsubsection{Binary image}

The binarization process of the image is to set the gray level of the points on the image to 0 or 255 , that is, to change the entire image screen to only black and white. That is, a grayscale image of 256 brightness levels is selected through an appropriate threshold to obtain a binary image that can still reflect the overall and local characteristics of the image. When the image is further processed, the collective nature of the image is only related to the position of the point where the pixel value is 0 or 255 . It does not involve the multi-level value of the pixel, which makes processing simple, and the amount of data processing and compression is small. Utilizing the feature that the license plate has a uniform gray value in the interior, the image is binarized to facilitate the positioning and segmentation of the license plate in the next step. The gray value is 0 for black, and the gray value is 255 for white. When writing the program, use the im $2 \mathrm{bw}$ function to binarize the image.

Choosing an appropriate threshold is the key to the binarization of the image, and it has a decisive influence on the effect of binarization. Common threshold selection methods include the histogram method, iterative method, and maximum inter-class variance method (OTSU method). We chose the method of maximum inter-class variance (OTSU). It is an automatic threshold selection method, which is widely used because it has the advantages of simplicity, strong selfadaptation, and good segmentation effect. The threshold segmentation method of the maximum inter-class variance method is: given an initial threshold, and then iteratively divide the image into two parts, the foreground and the background.

The calculation algorithm is as follows:

The total average gradient of the image is

$$
\mu=\omega_{0} \times \mu_{0}+\omega_{1} \times \mu_{1}
$$

Inter-class variance is

$$
L=\omega_{0}\left(\mu_{0}-\mu\right)^{2}+\omega_{1}\left(\mu_{1}-\mu\right)^{2}
$$

Where, $\omega_{0}$ and $\omega_{1}$ are the proportion of foreground and background pixels; $\mu_{0}$ and $\mu_{1}$ are the average gradient of foreground and background pixels.

\subsection{License Plate Positioning}

\subsubsection{Edge detection}

The edge detection of digital images is a very important basis for image analysis such as image segmentation, target area recognition, and area shape extraction. Edge is an important feature of an image. The first step in image understanding and analysis is often edge detection. The edges appear in the form of discontinuous local features of the image, that is, the most significant changes in the local brightness of the image, such as sudden changes in gray values, sudden changes in color, and sudden changes in texture structure. At the same time, the edges are also the boundaries of different regions. The edge of the image has two characteristics of direction and amplitude. Usually, the gray change along the edge is gentle, and the gray change perpendicular to the edge is sharp. Due to the edge is the place where the gray level changes most sharply in the image, traditional edge detection uses this feature to determine the edge point by calculating the gradient value of the pixel in the image. The purpose of edge detection is to find a set of pixels with sharp changes in brightness in the image, which often appear as contours. If the edges in the image can be accurately measured and located, it means that the actual object can be located and measured[7]

In the edge detection algorithm, due to the Sobel operator has a smoothing effect on noise, it can filter out some noise, provide accurate edge direction information, and is simple and effective. Therefore, this article uses Sobel operator for edge detection and uses the edge () function to complete the edge detection.

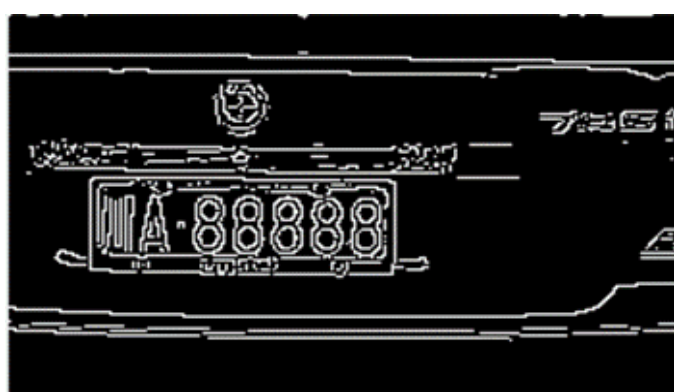

Fig 6: Image after edge detection

\subsubsection{Morphological processing}

Morphological operations are a series of image processing operations based on shapes. Morphological processing can realize the functions of eliminating noise, segmenting independent image elements, connecting adjacent elements in the image, and finding obvious maximum or minimum value regions in the image.

\subsubsection{Corrosion}

The role of corrosion is to eliminate object boundary points. Corrosion can remove objects smaller than the structural elements. In this way, selecting different structural elements can remove objects of different sizes. If there is a small communication between two objects, then when the structural elements are large enough, the two objects can be separated by the corrosion operation. Therefore, the image of the etched edge is found by the etch. 


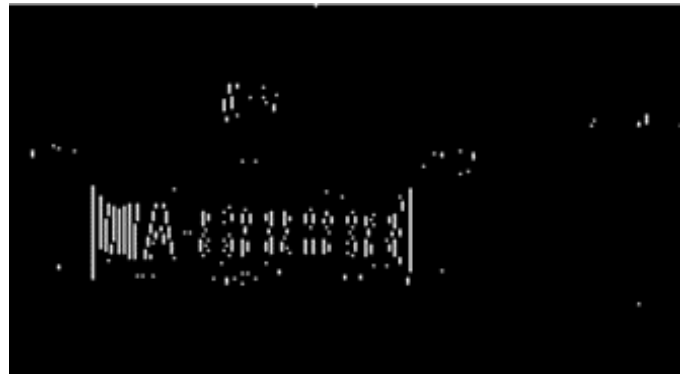

Fig 7: Post-corrosion image

\subsubsection{Cluster filling}

Using rectangular structural elements, clustering and filling are performed after corroding the image to obtain a more accurate and complete license plate position.

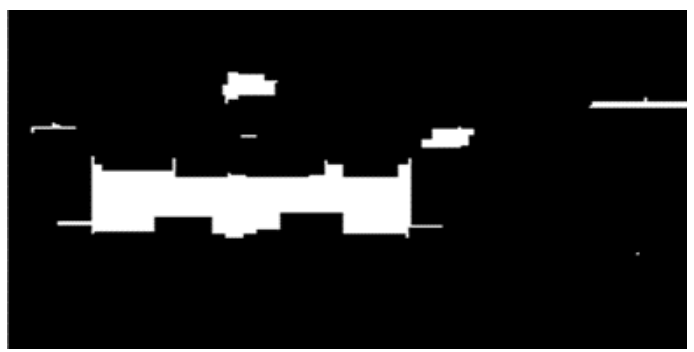

Fig 8: Cluster-filled image

\subsubsection{Morphological filtering}

In the license plate image segmented by the above method, there are target objects, background, and noise, that is, some of the obtained connected regions are invalid and ineligible. Therefore, conditions need to be set for screening to determine specific license plate locations. We use the bwareaopen () function to filter connected areas, remove small objects that do not meet the characteristics of the license plate, and locate the license plate area, that is, remove all connected objects with less than 2000 pixels from the binary image.

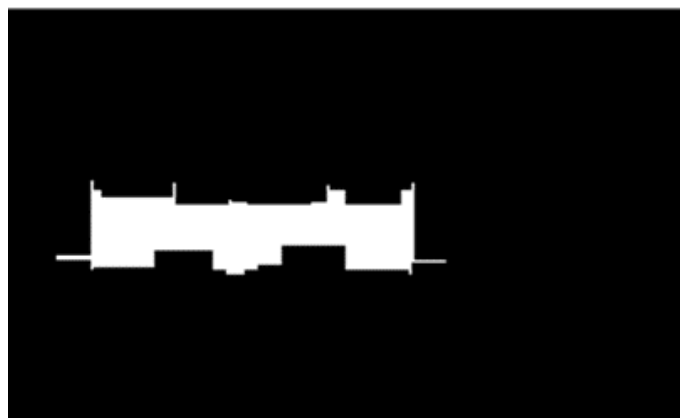

Fig 9: Morphological filtered image

\subsection{Character Segmentation}

\subsubsection{Uniform license plate background}

According to the prior information of the vehicle license plate, the license plate can be divided into four categories according to the background color and character color of the license plate. After graying, it can be divided into two categories according to the color: black on white and white on black. In order to facilitate subsequent license plate processing, the background color of the license plate should be unified, and the pictures with black characters on white background should be reversed to be unified into pictures with white characters on black background.

According to the observation of license plate images, most of the images have more backgrounds and smaller targets. According to the characteristics of this observation, the target can be distinguished from the background by counting the ratio of pixels of different colors. Assuming that the proportion of character pixels in the license plate image is $\mathrm{m}$ and the proportion of background pixels is $\mathrm{n}$, then $0<\mathrm{m}<\mathrm{n}$ $<1$. Therefore, the background color of the license plate can be determined by counting the proportion of the white pixels in the binarized license plate image. If $m \leq 50 \%$, the license plate image is binarized to a black background and white characters, no color inversion is required. If $\mathrm{m}>50 \%$, the license plate image will have a white background and black characters after binarization. The image needs to be reversed, that is, the white pixel point 1 in the license plate binarized image is changed to black pixel point 0 , and the black pixels are changed. Point 0 becomes white pixel point 1 . After color inversion processing, all license plates are unified into white characters with black background.

\subsubsection{Remove border}

We use the method of scanning from left to right and from top to bottom, keeping the connected domain size of the first white pixel point to the last white pixel point to achieve the purpose of removing the border. After removing the border, as shown.

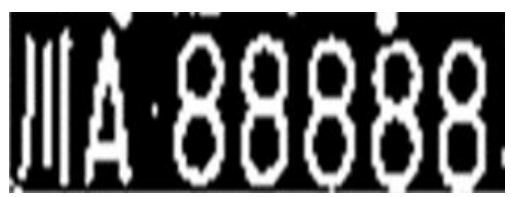

Fig 10: Image after removing the border

\subsubsection{Split image}

When recognizing a vehicle license plate, the number or English characters contained in the license plate are finally extracted. Therefore, after extracting the license plate picture from the photo, the numbers and English characters on the photo should be extracted and segmented separately. Character segmentation can usually choose the method of connected fields. We segment according to the characteristics of the license plate character arrangement position: the license plate has seven characters, the first is a Chinese character, the second is a letter, the remaining five are numbers and letters, and the second character There is a dot (counting half a character) between it and the third character. The width of the characters is the same, so a single character can be divided accurately based on the width of the characters.

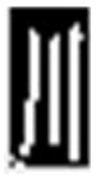

\subsection{Classification Recognition}

\subsubsection{Feature extraction}

Features are the most relevant information extracted from the original data that is relevant to the classification. This information minimizes intra-class variance and maximizes inter-class variance. In the recognition method based on BP neural network, feature extraction is very important, which basically determines the correct rate of recognition. The gray feature is the basic feature for judging each character. Therefore, the gray feature of the image is selected to identify the character. 
Take the recognition character $\mathrm{A}$ as an example:

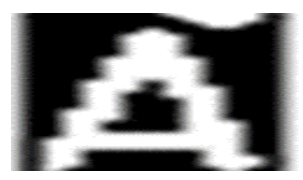

Fig 12: License plate character A

Fig 12 is normalized into a $20 * 20$-pixel bitmap, and each pixel bit is 0 or 1 to form 400 grayscale feature values of the network.

\subsubsection{Network structure design}

According to the "People's Republic of China Motor Vehicle License Plates" and related standards, the first character of license plate number is Chinese characters, which represent the names of provinces, autonomous regions or municipalities directly under the Central Government, with a total of 31 . The second to seventh characters can be English uppercase characters or Arabic numerals, which are used to distinguish different information of other vehicles. It should be noted that I and $\mathrm{O}$ of 26 uppercase English characters cannot be used. 09. Therefore, there are 34 characters in letters and numbers[10].

According to the characteristics of license plates, in order to reduce the processing amount of a single network, two corresponding BP neural networks are set up, which are the Chinese character network and the alphanumeric network. After dividing the license plate, the first character is sent to the Chinese character network through feature extraction, and the second to seventh characters are sent to the alphanumeric network. Through such a network design, it is avoided to compare the extracted features of Chinese characters with the features of letters and numbers, which is beneficial to improving the recognition rate and accuracy[14].

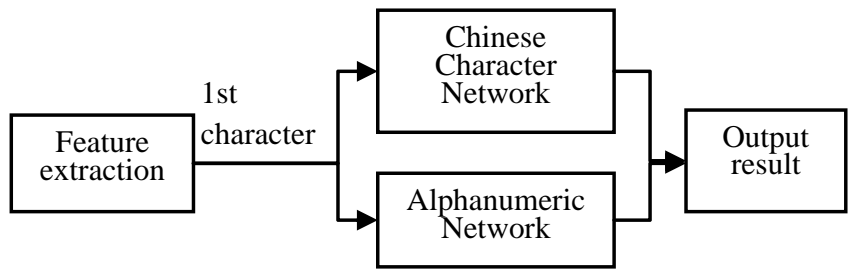

Fig 13: Neural network structure design

Both neural networks select the eigenvalues of each character image to construct a feature vector, that is, arrange the normalized grayscale bitmap into a single column vector of 400 rows * 1 columns as the input of the BP neural network.

\subsubsection{Parameter settings}

a. Number of neurons in the input layer

In general design, the number of neurons in the input layer is determined according to the feature dimension obtained by the feature extraction method used for characters. The feature extraction method used in this paper has 400 feature values, so the number of input neurons is 400 .

\section{b. Number of neurons in the output layer}

The number of neurons in the output layer is selected as the type of recognition. Therefore, the number of output neurons of the Chinese character network is 31 , and the number of output neurons of the alphanumeric network is 34 .

c. Number of neurons in the hidden layer

When simulating the relationship between input data and output data through a neural network, the number of neurons in the hidden layer is very important. The number of hidden layer neurons will directly affect the learning ability of the neural network. When the number of neurons is too small, although the learning time of the network is short, it will cause the network's mapping capacity to be insufficient and cannot achieve a good learning effect[13]. The weights are adjusted back and forth in multiple error back-propagation but still cannot reach the minimum error, and the training accuracy is not high; When the number of neurons is too large, the learning ability of the network is enhanced, but the learning time of the network is longer, and the storage space required by the network will also increase; In addition, too many or too few neurons may reduce the network's inductive ability, and then cause a large deviation in data output.

At present, there are several reference empirical formulas for determining the number of nodes in the hidden layer[12]:

$$
\begin{gathered}
l=2 n+1 \\
l=\sqrt{(m+n)}+a \\
l=\log _{2} n
\end{gathered}
$$

Where, $l$ is the number of nodes in the hidden layer; $n$ is the number of nodes in the input layer; $m$ is the number of nodes in the output layer; $a$ is a constant between $[0,10]$.

In practical problems, when selecting the number of nodes in the hidden layer, we solve the reference empirical formula to obtain an interval $[a, b]$ about the number of nodes in the hidden layer, and then use the trial and error method to determine the optimal number of nodes. After many experiments and comparisons, we select $\mathrm{N}=300$. At this time, the BP neural network has reached a higher accuracy.

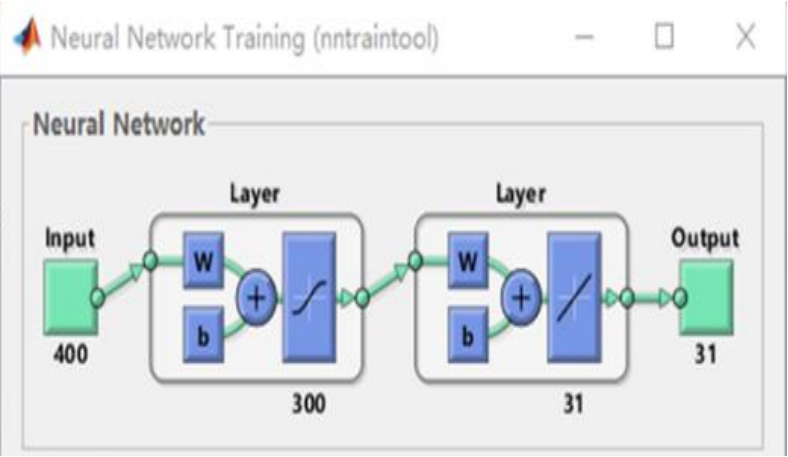

Fig 14: Chinese character network parameter settings

\section{Neural Network Training (nntraintool)}

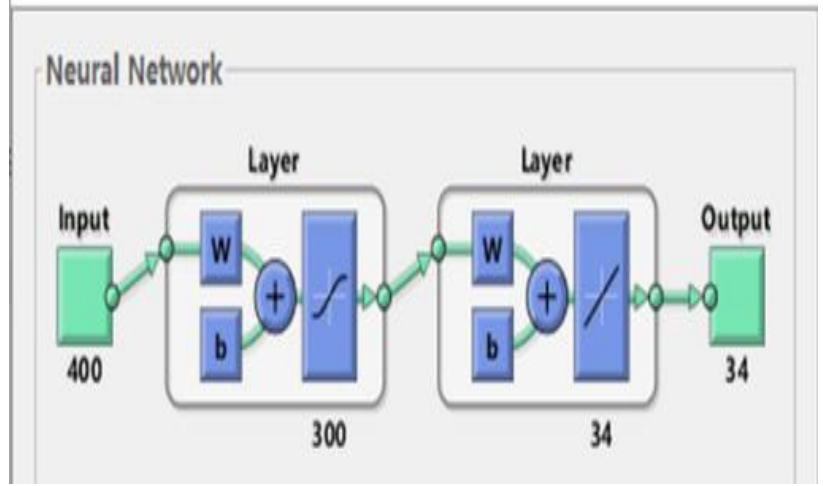

Fig 15: Alphanumeric network parameter settings 


\section{RESULTS}

\subsection{Neural Network Performance Analysis}

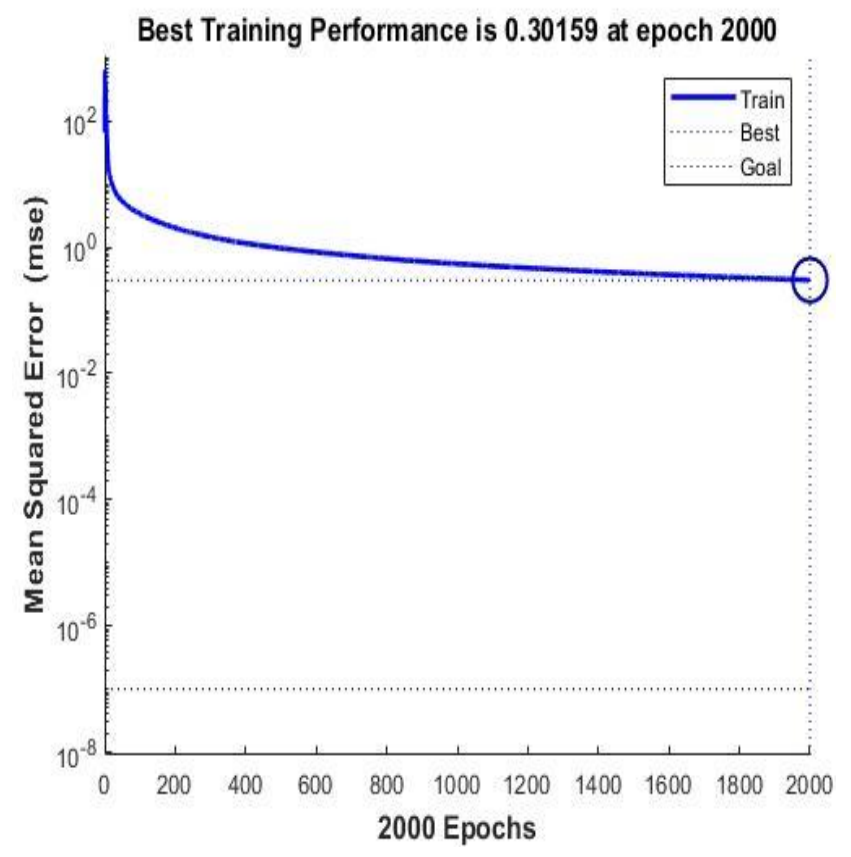

Fig 16: Chinese character network training results

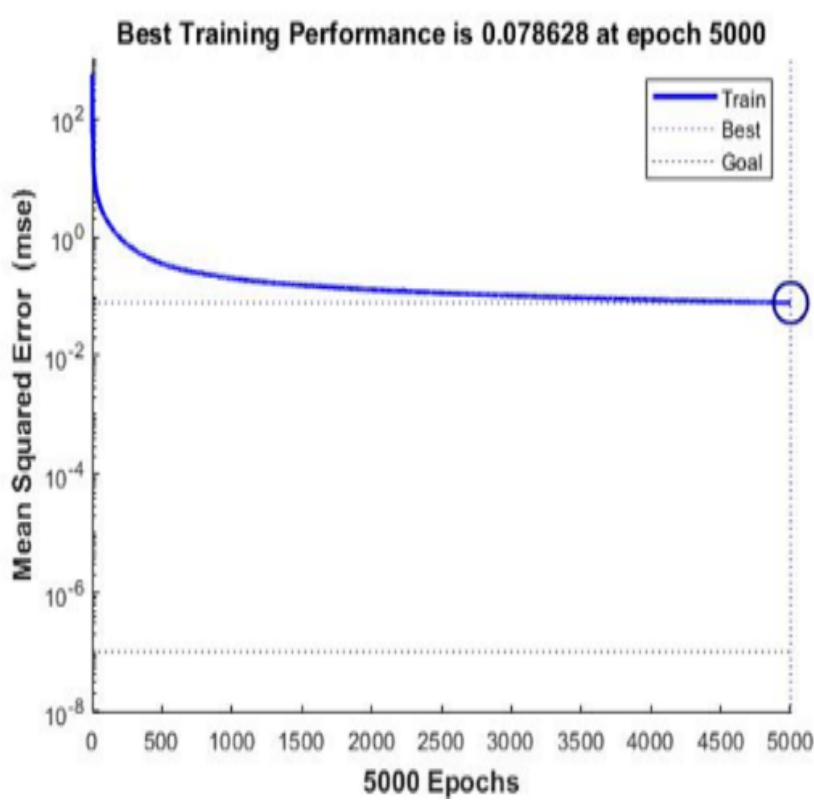

Fig 17: Alphanumeric network training results

By comparing and analyzing the settings of the network parameters in the system, the optimal network parameters can be obtained, and the data can show that the performance of each network is optimal at this time.

\subsection{Recognition Result}

We use the structure design and network parameters of the neural network for setting, and use MATLAB to simulate it. For Chinese character network, each character picks up about 140 training samples on average. Take the first 400 lines, that is, the feature value of each sample as input, and normalize the 401th line, that is, the template corresponding to the sample, as the target. The total number of training samples brought in is 4440 . For alphanumeric network, each character selects about 700 training samples on average. Take the first 400 lines, that is, the feature value of each sample as input, and normalize the 401th line, that is, the template corresponding to the sample, as the target. The total number of training samples brought in is 24652 .

After successful training, we designed the GUI interface to make the license plate recognition process more intuitive, and to achieve the purpose of making the license plate recognition system more practical and convenient.

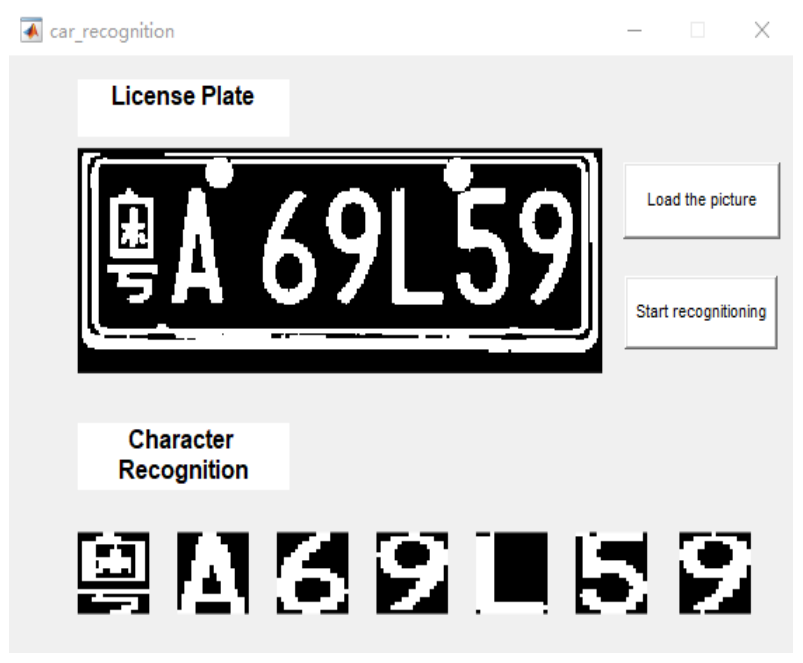

Fig 18:Recognition process

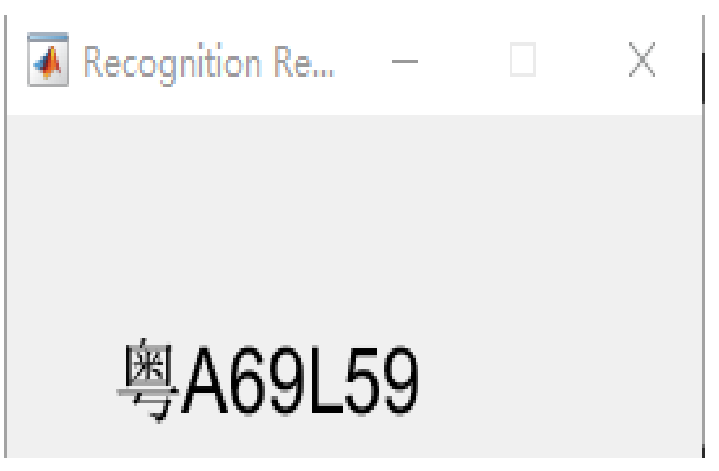

Fig 19: Recognition result

\section{CONCLUSION}

1. Based on the improved neural network, we designed a license plate recognition system. The system has four modules, namely: image preprocessing, license plate positioning, character segmentation and classification recognition.

2. We briefly introduced the process, steps and their importance in image preprocessing, discussed the common methods of license plate positioning and character segmentation, and detailed the methods used in the research of this subject, and finally gave the processed the result of.

3 . We conducted a key analysis of the neural network used in the research of this subject, and designed two corresponding networks for identifying Chinese characters and numbers according to the specificity of China's license plates, and analyzed the settings of each network parameter. On this basis, train the network, use the trained network for license plate recognition, get the license plate recognition results, and simulate the system through MATLAB. 


\section{REFERENCES}

[1] Guo, K.X., Wang, G.W. ( 2010 ). License plate location algorithm based on dynamic HSV threshold. Big technology. Technology World:116-119.

[2] Wu, Y.J., Liu, S.X., Wang. (2013). License plate location method based on texture and color. IEEE 、 IEEE Beijing Section. Proceedings of 2013 IEEE 4th International Conference on Software Engineering and Service Science. IEEE、 IEEE Beijing Section: IEEE BEIJING SECTION.

[3] Yang, C.X., Zhang, Z.H. (2019). Research on License Plate Recognition Algorithm Based on Particle Swarm Optimization. Journal of Guizhou University (Natural Science Edition), 36(06),42-45.

[4] Li, Y.W., Tong, J.Y., Hu, X.D., Zhang, C.B., Li, H.L. (2018). Automatic license plate recognition based on BP neural network algorithm. Industrial control computer.

[5] Wang, X.F. (2019). Research on License Plate Detection and Recognition Based on Deep Learning. Nanjing University of Posts and Telecommunications.

[6] Wu, S.W. (2019). Study the main technologies and application prospects of artificial intelligence in the field of transportation. Communication World.

[7] Yu, X.Y., Zhang, Y., Xu, Y. (2020). A Canny algorithm with improved adaptive threshold. Machinery and Electronics.

[8] Huang, Y.G., Zhang, Y.S. (2020). Handwritten digit recognition system based on BP neural network. Electromechanical Engineering Technology.
[9] Cheng, D., Lu, H.C., Gao, W.G. (2019). License Plate Location Algorithm Based on Improved Canny Operator Edge Detection and Mathematical Morphology. Journal of Heilongjiang Institute of Technology.

[10] Ministry of Public Security of the People's Republic of China. (2014).GA36-2014 The People's Republic of China Motor Vehicle License Plate Public Safety Industry Standard.

[11] Qiu, W. (2018). Research on Character Recognition of License Plate Based on GABP Neural Network. Jilin University.

[12] Yin, G.G. (2019). Study on the Number of Hidden Layer Nodes in Neural Network. Dalian University of Technology.

[13] Wang, R.B., Xu, H.Y., Li, B., Feng, Y. (2017). Research on the Method of Determining the Number of Hidden Layer Nodes in BP Neural Network. Computer Technology and Development.

[14] Shen, J. (2013). Research on License Plate Recognition Technology Based on BP Neural Network. Hebei University of Technology.

[15] Yang, W.L., Xiao, C.W. (2019). An Algorithm Implementation of BP Network with Adaptive Momentum Factor. Computer and Digital Engineering.

[16] Chen, Z., Ding, S., Wang, J.L. (2015). BP Neural Network Model Prediction and Analysis of National Private Vehicle Ownership_-BP Method Based on Combination of Additional Momentum and Adaptive Learning Rate. Journal of Xi'an University of Finance and Economic. 